\title{
Some Results for Fuzzy Maps Under Nonexpansive Type Condition
}

\author{
Sweetee Mishra, R.K. Namdeo and Brian Fisher \\ Abstract. In this paper, we have proved some results for fuzzy maps \\ satisfying non-expansive type condition.
}

\section{INTRODUCTION}

A mapping $T: X \rightarrow X$ is called non-expansive if its Lipschitz constant $k(T)$ does not exceed 1 . Thus, this class of mappings includes the contraction and strictly contractive mappings; moreover it contains all isometries (including the identity).

A map $T: X \rightarrow X$ is said to be non-expansive if

$$
d(T x, T y) \leq d(x, y), \text { forall } x, y \in X .
$$

Ciric studied the following non-expansive type condition in his paper [1] and [2] for a self map, $T$ of $X$ :

$$
\begin{aligned}
& d(T x, T y) \leq a \max \{d(x, y), d(x, T x), d(y, T y)\} \\
&+b \max \{d(x, T x), d(y, T y)\} \\
&+c[d(x, T y)+d(y, T x)] \\
& d(T x, T y) \leq a d(x, y)+b \max \{d(x, T x), d(y, T y)\}+c[d(x, T y), d(y, T x)]
\end{aligned}
$$

for all $x, y \in X$, where $a, b, c \geq 0$ such that $a+b+2 c=1$.

The fuzzy set was introduced by L. Zadeh [9] in 1965. In this paper we shall use the terminology and notation of Heilpern [3]. Heilpern gave some fundamental results related to fuzzy map. Since that time a substantial literature has developed on this subject. In some earlier work Rhoades and Bruce Watson $[7,8]$ proved several fixed point theorems involving a very general contractive condition, for fuzzy maps on complete linear metric space.

Definition 1. A fuzzy set $A$ in complete metric space $X$ is a function from $X$ into $[0,1]$. If $x \in X$, the function value $A(x)$ is called the grade of member

1991 Mathematics Subject Classification. Primary:

Key words and phrases. Fuzzy maps, Common fixed point, Non-expansive map. 
of $x$ in $A$. The $\alpha$-level set of $A$, denoted by

$$
\begin{aligned}
& A_{\alpha}=\{x: A(x) \geq \alpha\} \quad \text { if } \quad \alpha \in(0,1], \\
& A_{0}=\{x: A(x)>0\} .
\end{aligned}
$$

Definition 2. A fuzzy set $A$ is said to be an approximate quantity iff $A_{\alpha}$ is compact and convex for each $\alpha \in[0,1]$, and $\sup _{x \in X} A(x)=1$.

When $A$ is an approximate quantity and $A\left(x_{0}\right)=1$ for some $x_{0} \in X, A$ is identified with an approximation of $x_{0}$.

The collection of all fuzzy sets in $X$ is denoted by $F(X)$ and $W(X)$ is the sub-collection of all approximate quantities.

Definition 3. Let $A, B \in W(X), \alpha \in[0,1]$. Then

$$
\begin{aligned}
D_{\alpha}(A, B) & =\inf _{x \in A_{\alpha}, y \in B_{\alpha}} d(x, y), \\
D(A, B) & =\sup _{\alpha} D_{\alpha}(A, B), \\
H_{\alpha}(A, B) & =\operatorname{dist}\left(A_{\alpha}, B_{\alpha}\right),
\end{aligned}
$$

where "dist" is the Hausdorff distance.

Definition 4. Let $A, B \in W(X)$, then $A$ is said to be more accurate than $B$, denoted by $A \subset B$ iff $A(x) \leq B(x)$ for each $x \in X$.

The relation " $\subset$ " induces a partial ordering on the family $W(X)$.

Definition 5. Let $X$ and $Y$ be two complete linear metric spaces. $F$ is called a fuzzy mapping if and only if $F$ is a mapping from the set $X$ into $W(Y)$.

A fuzzy mapping $F$ is a fuzzy subset of $X \times Y$ with membership function $F(x, y)$. The function value $F(x, y)$ is the grade of membership of $y$ in $F(x)$. Each fuzzy mapping is a set valued mapping.

Lee [4] proved the following Lemma.

Lemma 1. Let $(X, d)$ be a complete linear metric space, $F$ is a fuzzy map from $X$ into $W(X)$ and $x_{0} \in X$ then there exists an $x_{1} \in X$ such that $\left\{x_{1}\right\} \subset F\left(x_{0}\right)$.

The following two lemmas are due to Heilpern [3].

Lemma 2. Let $A, B \in W(X), \alpha \in[0,1]$, and $D_{\alpha}(A, B)=\inf _{x \in A_{\alpha}, y \in B_{\alpha}} d(x, y)$, where $A_{\alpha}=\{x: A(x) \geq \alpha\}$, then $D_{\alpha}(x, A) \leq d(x, y)+D_{\alpha}(y, A)$ for each $x, y \in X$.

Lemma 3. Let $H_{\alpha}(A, B)=\operatorname{dist}\left(A_{\alpha}, B_{\alpha}\right)$ where "dist" is the Hausdorff distance. If $\left\{x_{0}\right\} \subset A$ then $D_{\alpha}\left(x_{0}, B\right) \leq H_{\alpha}(A, B)$ for each $B \in W(X)$.

Rhoades [5] proved the following common fixed point theorem involving a very general contractive condition, for fuzzy maps on complete linear metric space. 
Theorem A. Let $(X, d)$ be complete linear metric space and let $F, G$ be fuzzy mappings from $X$ into $W(X)$ satisfying

$$
H(F x, G y) \leq Q(m(x, y)) \text { of all } x, y \text { in } X
$$

where

$$
\begin{gathered}
m(x, y)=\max \left\{d(x, y), D_{\alpha}(x, F x), D_{\alpha}(y, G y),\right. \\
\left.\frac{1}{2}\left[D_{\alpha}(x, G y)+D_{\alpha}(y, F x)\right]\right\},
\end{gathered}
$$

$Q$ is a real-valued function defined on $D$, the closure of the range of $d$, satisfying the following three conditions:

(a) $0<Q(s)<s$ for each $s \in D \backslash\{0\}$ and $Q(0)=0$,

(b) $Q$ is non-decreasing on $D$, and

(c) $g(s)=s / s-Q(s)$ is non-increasing on $D \backslash\{0\}$.

Then there exists a point $z$ in $X$, such that $\{z\} \subset F z \cap G z$.

We have proved the following common fixed point theorem satisfying nonexpansive condition, for fuzzy maps on complete linear metric space.

Theorem 1. Let $(X, d)$ be a complete linear metric space. $F, G$ are fuzzy mappings from $X$ into $W(X), T$ is a self-map of $X$, satisfying

$$
\begin{aligned}
H(F x, G y) \leq & a \max \left\{d(T x, T y), D_{\alpha}(T x, F x), D_{\alpha}(T y, G y),\right. \\
& \left.\frac{1}{2}\left[D_{\alpha}(T x, G y)+D_{\alpha}(T y, F x)\right]\right\} \\
+ & b \max \left\{D_{\alpha}(T x, F x), D_{\alpha}(T y, G y)\right\} \\
+ & c\left[D_{\alpha}(T x, G y)+D_{\alpha}(T y, F x)\right],
\end{aligned}
$$

where $a, b, c$ are non-negative real numbers such that $a+b+2 c=1$.

If $T$ is continuous, $T$ is weakly commutes with $S$ and $T$ and there exist a sequence which is asymptotically $F$-regular and $G$-regular with respect to $T$, then there exists a point $z$ in $X$, which is a common fixed point of maps $F$, $G, T$.

Proof. Let $x_{0} \in X$, then by Lemma 1 , we can choose $T x_{1} \in X$ such that $\left\{T x_{1}\right\} \subset F x_{0}$. Choose $x_{2}$ such that $d\left(T x_{1}, T x_{2}\right) \leq H\left(F x_{0}, G x_{1}\right)$, continuing the process we obtain a sequence $\left\{T x_{n}\right\}$ such that $\left\{T x_{2 n+1}\right\} \subset F x_{2 n}$, $\left\{T x_{2 n+2}\right\} \subset G x_{2 n+1}$ and $d\left(T x_{2 n+1}, x_{2 n+2}\right) \leq H\left(F x_{2 n}, G x_{2 n+1}\right)$, where $n=$ $1,2,3 \ldots$ 
Applying (2) and using triangle inequality, we have,

$$
\begin{aligned}
d( & \left.T x_{2 n}, T x_{2 n+1}\right) \leq H\left(F x_{2 n-1}, G x_{2 n}\right) \\
\leq & a \max \left\{d\left(T x_{2 n-1}, T x_{2 n}\right), D_{\alpha}\left(T x_{2 n-1}, F x_{2 n-1}\right),\right. \\
& \left.D_{\alpha}\left(T x_{2 n}, G x_{2 n}\right), \frac{1}{2}\left[D_{\alpha}\left(T x_{2 n-1}, G x_{2 n}\right)+D_{\alpha}\left(T x_{2 n}, F x_{2 n-1}\right)\right]\right\} \\
+ & b \max \left\{D_{\alpha}\left(T x_{2 n-1}, F x_{2 n-1}\right), D_{\alpha}\left(T x_{2 n}, G x_{2 n}\right)\right\} \\
+ & c\left[D_{\alpha}\left(T x_{2 n-1}, G x_{2 n}\right)+D_{\alpha}\left(T x_{2 n}, F x_{2 n-1}\right)\right] \\
\leq & a \max \left\{d\left(T x_{2 n-1}, T x_{2 n}\right), d\left(T x_{2 n}, T x_{2 n+1}\right),\right. \\
& \left.\frac{1}{2}\left[d\left(T x_{2 n-1}, T x_{2 n}\right)+d\left(T x_{2 n}, T x_{2 n+1}\right)\right]\right\} \\
+ & b \max \left\{d\left(T x_{2 n-1}, T x_{2 n}\right)+d\left(T x_{2 n}, T x_{2 n+1}\right)\right\} \\
+ & c\left[d\left(T x_{2 n-1}, T x_{2 n}\right)+d\left(T x_{2 n}, T x_{2 n+1}\right)\right] \\
\leq & (a+b) \max \left\{d\left(T x_{2 n-1}, T x_{2 n}\right), d\left(T x_{2 n}, T x_{2 n+1}\right)\right\} \\
+ & c\left[d\left(T x_{2 n-1}, T x_{2 n}\right)+d\left(T x_{2 n}, T x_{2 n+1}\right)\right] .
\end{aligned}
$$

If $d\left(T x_{2 n}, T x_{2 n+1}\right)>d\left(T x_{2 n-1}, T x_{2 n}\right)$ for some $n$, then we have,

$$
\begin{aligned}
d\left(T x_{2 n}, T x_{2 n+1}\right) & \leq(a+b+2 c) d\left(T x_{2 n}, T x_{2 n+1}\right) \\
& =d\left(T x_{2 n}, T x_{2 n+1}\right)
\end{aligned}
$$

a contradiction. Thus $d\left(T x_{2 n}, T x_{2 n+1}\right) \leq d\left(T x_{2 n-1}, T x_{2 n}\right)$.

Hence, for all positive integers $n$,

$$
d\left(T x_{2 n}, T x_{2 n+1}\right) \leq d\left(T x_{0}, T x_{1}\right) .
$$

Again applying (2) and using (3) we get

$$
\begin{aligned}
d( & \left.T x_{2}, T x_{3}\right) \leq a \max \left\{d\left(T x_{1}, T x_{2}\right), d\left(T x_{2}, T x_{3}\right), d\left(T x_{1}, T x_{2}\right)\right. \\
& \left.\quad \frac{1}{2}\left[d\left(T x_{2}, T x_{2}\right)+d\left(T x_{1}, T x_{3}\right)\right]\right\} \\
& +b \max \left\{d\left(T x_{1}, T x_{2}\right), d\left(T x_{2}, T x_{3}\right)\right\} \\
\quad+ & c\left[d\left(T x_{1}, T x_{3}\right)+d\left(T x_{2}, T x_{2}\right)\right] \\
\quad \leq & a \max \left\{d\left(T x_{0}, T x_{1}\right), d\left(T x_{0}, T x_{1}\right), d\left(T x_{0}, T x_{1}\right), \frac{1}{2} d\left(T x_{1}, T x_{3}\right)\right\} \\
\quad+ & b \max \left\{d\left(T x_{0}, T x_{1}\right), d\left(T x_{0}, T x_{1}\right)\right\}+c d\left(T x_{1}, T x_{3}\right)
\end{aligned}
$$


Applying (2) again and using (3) we have

$$
\begin{aligned}
d\left(T x_{1}, T x_{3}\right) \leq & a \max \left\{d\left(T x_{0}, T x_{1}\right), d\left(T x_{2}, T x_{3}\right), d\left(T x_{0}, T x_{2}\right),\right. \\
& \left.\frac{1}{2}\left[d\left(T x_{0}, T x_{3}\right)+d\left(T x_{2}, T x_{1}\right)\right]\right\} \\
+ & b \max \left\{d\left(T x_{0}, T x_{1}\right), d\left(T x_{2}, T x_{3}\right)\right\} \\
+ & c\left[d\left(T x_{0}, T x_{3}\right)+d\left(T x_{2}, T x_{1}\right)\right] \\
\leq & (2-b) d\left(T x_{0}, T x_{1}\right) .
\end{aligned}
$$

Using (4) and (5), we get

$$
\begin{aligned}
d\left(T x_{2}, T x_{3}\right) & \leq a d\left(T x_{0}, T x_{1}\right)+b d\left(T x_{0}, T x_{1}\right)+(2 c-b c) d\left(T x_{0}, T x_{1}\right) \\
& \leq(1-b c) d\left(T x_{0}, T x_{1}\right) .
\end{aligned}
$$

It is easy to show that

$$
d\left(T x_{n+1}, T x_{n}\right) \leq(1-b c)^{[n / 2]} d\left(T x_{0}, T x_{1}\right),
$$

where $[n / 2]$ means the greatest integer not exceeding $n / 2$.

We conclude that $\left\{T x_{n}\right\}$ is Cauchy sequence. Since $X$ is complete, $\left\{T x_{n}\right\}$ is convergent to the point $z$ (say).

Since $\alpha \in[0,1]$ then using Lemmas 2, 3 and (2) we have

$$
\begin{aligned}
D_{\alpha}(T z, F z) & \left.\leq d\left(T z, G T x_{n}\right)\right)+D_{\alpha}\left(G T x_{n}, F z\right) \\
& \leq d\left(T z, G T x_{n}\right)+H_{\alpha}\left(F z, G T x_{n}\right) \\
& \leq d\left(T z, G T x_{n}\right)+H\left(F z, G T x_{n}\right) .
\end{aligned}
$$

Taking the limit $n$ tends to infinity we get

$$
D_{\alpha}(T z, F z) \leq \lim _{n \rightarrow \infty} H\left(F z, G T x_{n}\right) \leq \lim _{n \rightarrow \infty} H\left(F z, G T x_{n}\right)
$$

Again using (2) we have

$$
\begin{aligned}
H\left(F z, G T x_{n}\right) \leq & a \max \left\{d\left(T z, T T x_{n}\right), D_{\alpha}\left(T z, F T x_{n}\right), D_{\alpha}\left(T T x_{n}, G T x_{n}\right),\right. \\
& \left.\frac{1}{2}\left[D_{\alpha}\left(T z, G T x_{n}\right)+D_{\alpha}\left(T T x_{n}, F z\right)\right]\right\} \\
+ & b \max \left\{D_{\alpha}(T z, F z), D_{\alpha}\left(T T x_{n}, G T x_{n}\right)\right\} \\
+ & c\left[D_{\alpha}\left(T z, G T x_{n}\right)+D_{\alpha}\left(T T x_{n}, F z\right)\right] .
\end{aligned}
$$

Letting $n$ tend to infinity, we have

$$
\begin{aligned}
\lim _{n \rightarrow \infty} H\left(F z, G T x_{n}\right) \leq & a \max \left\{d(T z, T z), d(T z, F z), D_{\alpha}(T z, G z),\right. \\
& \left.\frac{1}{2}[d(T z, G z)+d(T z, F z)]\right\} \\
+ & b \max \{d(T z, F z), d(T z, G z)\} \\
+ & c[d(T z, G z)+d(T z, F z)]
\end{aligned}
$$




$$
\begin{aligned}
\lim _{n \rightarrow \infty} H\left(F z, G T x_{n}\right) & \leq(a+b+2 c) \max \{d(T z, F z), d(T z, G z)\} \\
& =d(T z, F z) .
\end{aligned}
$$

Using (6) and (7) we have

$$
D_{\alpha}(T z, F z) \leq d(T z, F z),
$$

a contradiction. Hence we must have $D_{\alpha}(T z, F z)=0$. Since $\alpha$ is arbitrary number in $[0,1]$. It follows that $D(T z, F z)=0$, which implies that $T z=F z$ Similarly it can be shown that $T z=G z$.

$$
\begin{aligned}
H\left(F x_{n}, G T x_{n}\right) \leq & a \max \left\{d\left(T x_{n}, T T x_{n}\right), D_{\alpha}\left(T x_{n}, F x_{n}\right), D_{\alpha}\left(T T x_{n}, G T x_{n}\right),\right. \\
& \left.\frac{1}{2}\left[D_{\alpha}\left(T x_{n}, G T x_{n}\right)+D_{\alpha}\left(T T x_{n}, F x_{n}\right)\right]\right\} \\
+ & b \max \left\{D_{\alpha}\left(T x_{n}, F x_{n}\right), D_{\alpha}\left(T T x_{n}, G T x_{n}\right)\right\} \\
+ & c\left[D_{\alpha}\left(T x_{n}, G T x_{n}\right)+D_{\alpha}\left(T T x_{n}, F x_{n}\right)\right]
\end{aligned}
$$

Letting $n$ tend to infinity and supposing $T$ is continuous, $T$ weakly commutes with $S$ and $T$ and there exist a sequence which is asymptotically $F$-regular and $G$-regular with respect to $T$, than we have

$$
\begin{aligned}
& \leq a d(z, T z)+2 c d(z, T z) \\
& d(z, T z) \leq(1-b) d(z, T z),
\end{aligned}
$$

which implies $z=T z$.

Hence $z$ is a common fixed point of maps $G, F, T$.

Corollary 1. Let $(X, d)$ be a complete linear metric space. $F, G$ are fuzzy mappings from $X$ into $W(X)$ satisfying

$$
\begin{aligned}
H(F x, G y) \leq & a \max \left\{d(x, y), D_{\alpha}(x, F x), D_{\alpha}(y, G y),\right. \\
& \left.\frac{1}{2}\left[D_{\alpha}(x, G y)+D_{\alpha}(y, F x)\right]\right\} \\
& b \max \left\{D_{\alpha}(x, F x), D_{\alpha}(y, G y)\right\} \\
+ & c\left[D_{\alpha}(x, G y)+D_{\alpha}(y, F x)\right]
\end{aligned}
$$

where $a, b, c$ are non-negative real numbers such that $a+b+2 c=1$.

Then there exists a point $z$ in $X$, which is a common fixed point of maps $F$ and $G$, i.e., $\{z\} \subset F z \cap G z$.

Proof. Taking $T$ is identity map of $X$ in Theorem 1 .

Rhoades [6], generalized the result of Theorem A for sequence of fuzzy maps on complete linear metric space. He proved the following theorem.

Theorem B. Let $g$ be a non-expansive self mapping of a complete linear metric space $(X, d)$. Let $\left\{F_{i}\right\}$ be a sequence of fuzzy mappings from $X$ into $W(X)$. For each pair of fuzzy mappings $F_{i}, F_{j}$ and for any $x \in X,\left\{u_{x}\right\} \subset$ 
$F_{i}(x)$, there exists a $\left\{v_{y}\right\} \subset F_{j}(y)$ for all $y \in X$ such that $D\left(\left\{u_{x}\right\},\left\{v_{y}\right\}\right) \leq$ $Q(m(x, y))$, where

$$
\begin{gathered}
m(x, y)=\max \left\{d\left(g(x), g\left(u_{x}\right)\right), d\left(g(y), g\left(v_{y}\right)\right), d(g(x), g(y)),\right. \\
\left.\frac{1}{2}\left[d\left(g(x), g\left(v_{y}\right)\right)+d\left(g(y), g\left(u_{x}\right)\right)\right]\right\}
\end{gathered}
$$

where $Q$ satisfying the conditions (a)-(c) of Theorem A. Then there exists $\{p\} \subset \bigcap_{i \in N} F_{i}(p)$.

We prove the result of above for common fixed point for sequence of fuzzy mappings of non-expansive condition.

Theorem 2. Let $g$ be a non-expansive self mapping of a complete linear metric space $(X, d)$. Let $\left\{F_{i}\right\}$ be a sequence of fuzzy mappings from $X$ into $W(X)$. For each pair of fuzzy mapping $F_{i}, F_{j}$ and for any $x \in X,\left\{u_{x}\right\} \subset$ $F_{i}(x)$, there exists $a\left\{v_{y}\right\} \subset F_{j}(y)$ for all $y \in X$ such that

$$
\begin{aligned}
D\left(\left\{u_{x}\right\},\left\{v_{y}\right\}\right) \leq & a \max \left\{d\left(g(x), g\left(u_{x}\right)\right), d\left(g(y), g\left(v_{y}\right)\right), d(g(x), g(y)),\right. \\
& \left.\frac{1}{2}\left[d\left(g(x), g\left(v_{y}\right)\right)+d\left(g(y), g\left(u_{x}\right)\right)\right]\right\} \\
+ & b \max \left\{d\left(g(x), g\left(u_{x}\right)\right), d\left(g(y), g\left(v_{y}\right)\right)\right\} \\
+ & c\left[d\left(g(x), g\left(v_{y}\right)\right)+d\left(g(y), g\left(u_{x}\right)\right)\right]
\end{aligned}
$$

where $a, b, c$ are non-negative real numbers such that $a+b+2 c=1$.

Then there exists $\{p\} \subset \bigcap_{i \in N} F_{i}(p)$, i.e., $p$ is a common fixed point of sequence of fuzzy mappings.

Proof. Let $x_{0} \in X$, then by Lemma 1 , we can choose $x_{1} \in X$ such that $\left\{x_{1}\right\} \subset F\left(x_{0}\right)$. Similarly for $x_{1} \in X$ we can choose $x_{2} \in X$ such that $\left\{x_{2}\right\} \subset F_{2}\left(x_{1}\right)$. In general, $\left\{x_{n+1}\right\} \subset F_{n+1}\left(x_{n}\right)$.

Applying (9) and using triangle inequality we have

$$
\begin{aligned}
d\left(x_{n}, x_{n+1}\right)= & D\left(\left\{x_{n}\right\},\left\{x_{n+1}\right\}\right) \\
\leq & a \max \left\{d\left(g\left(x_{n-1}\right), g\left(x_{n}\right)\right), d\left(g\left(x_{n}\right), g\left(x_{n+1}\right)\right), d\left(g\left(x_{n-1}\right), g\left(x_{n}\right)\right),\right. \\
& \left.\frac{1}{2}\left[d\left(g\left(x_{n}\right), g\left(x_{n}\right)\right)+d\left(g\left(x_{n-1}\right), g\left(x_{n+1}\right)\right)\right]\right\} \\
& +b \max \left\{d\left(g\left(x_{n-1}\right), g\left(x_{n}\right)\right), d\left(g\left(x_{n}\right), g\left(x_{n+1}\right)\right)\right\} \\
& +c\left[d\left(g\left(x_{n}\right), g\left(x_{n}\right)\right)+d\left(g\left(x_{n-1}\right), g\left(x_{n+1}\right)\right)\right] .
\end{aligned}
$$


Since $g$ is non-expansive and $D\left(\left\{x_{n}\right\},\left\{x_{n+1}\right\}\right)=d\left(x_{n}, x_{n+1}\right)$, we get

$$
\begin{aligned}
d\left(x_{n}, x_{n+1}\right) \leq & a \max \left\{d\left(x_{n-1}, x_{n}\right), d\left(x_{n}, x_{n+1}\right), d\left(x_{n-1}, x_{n}\right),\right. \\
& \left.\frac{1}{2}\left[d\left(x_{n}, x_{n}\right)+d\left(x_{n-1}, x_{n+1}\right)\right]\right\} \\
& +b \max \left\{d\left(x_{n-1}, x_{n}\right), d\left(x_{n}, x_{n+1}\right)\right\} \\
& +c\left[d\left(x_{n}, x_{n}\right)+d\left(x_{n-1}, x_{n+1}\right]\right. \\
\leq & a \max \left\{d\left(x_{n-1}, x_{n}\right), d\left(x_{n}, x_{n+1}\right), \frac{1}{2}\left[d\left(x_{n-1}, x_{n}\right)+d\left(x_{n}, x_{n+1}\right)\right]\right\} \\
& +b \max \left\{d\left(x_{n-1}, x_{n}\right), d\left(x_{n}, x_{n+1}\right)\right\} \\
& +c\left[d\left(x_{n-1}, x_{n}\right)+d\left(x_{n}, x_{n+1}\right)\right] .
\end{aligned}
$$

If $d\left(x_{n-1}, x_{n}\right)<d\left(x_{n}, x_{n+1}\right)$ for some $n$, then we have

$$
\begin{aligned}
d\left(x_{n}, x_{n+1}\right)< & a \max \left\{d\left(x_{n}, x_{n+1}\right), d\left(x_{n}, x_{n+1}\right), \frac{1}{2}\left[d\left(x_{n}, x_{n+1}\right)+d\left(x_{n}, x_{n+1}\right)\right]\right\} \\
& +b \max \left\{d\left(x_{n}, x_{n+1}\right), d\left(x_{n}, x_{n+1}\right)\right\} \\
& +c\left[d\left(x_{n}, x_{n+1}\right)+d\left(x_{n}, x_{n+1}\right)\right] \\
= & (a+b+2 c) d\left(x_{n}, x_{n+1}\right) \\
= & d\left(x_{n}, x_{n+1}\right)
\end{aligned}
$$

a contradiction. Thus $d\left(x_{n}, x_{n+1}\right) \leq d\left(x_{n-1}, x_{n}\right)$.

Hence, for all positive integers $n$

$$
d\left(x_{n}, x_{n+1}\right) \leq d\left(x_{0}, x_{1}\right) .
$$

Again applying (9) and using (10), we get

$$
\begin{aligned}
d\left(x_{2}, x_{3}\right)= & D\left(x_{2}, x_{3}\right) \leq a \max \left\{d\left(g\left(x_{1}\right), g\left(x_{2}\right)\right), d\left(g\left(x_{2}\right), g\left(x_{3}\right)\right), d\left(g\left(x_{1}\right), g\left(x_{2}\right)\right),\right. \\
& \left.\frac{1}{2}\left[d\left(g\left(x_{1}\right), g\left(x_{3}\right)\right)+d\left(g\left(x_{2}\right), g\left(x_{2}\right)\right)\right]\right\} \\
& +b \max \left\{d\left(g\left(x_{1}\right), g\left(x_{2}\right)\right), d\left(g\left(x_{2}\right), g\left(x_{3}\right)\right)\right\} \\
& +c\left[d\left(g\left(x_{1}\right), g\left(x_{3}\right)\right)+d\left(g\left(x_{2}\right), g\left(x_{2}\right)\right)\right] .
\end{aligned}
$$


Since $g$ is non-expansive, we have

$$
\begin{aligned}
d\left(x_{2}, x_{3}\right) \leq & a \max \left\{d\left(x_{1}, x_{2}\right), d\left(x_{2}, x_{3}\right), d\left(x_{1}, x_{2}\right)\right. \\
& \left.\frac{1}{2}\left[d\left(x_{1}, x_{3}\right)+d\left(x_{2}, x_{2}\right)\right]\right\} \\
& +b \max \left\{d\left(x_{1}, x_{2}\right), d\left(x_{2}, x_{3}\right)\right\}+c\left[d\left(x_{1}, x_{3}\right)+d\left(x_{2}, x_{2}\right)\right] \\
\leq & a \max \left\{d\left(x_{0}, x_{1}\right), d\left(x_{0}, x_{1}\right), d\left(x_{0}, x_{1}\right), \frac{1}{2} d\left(x_{1}, x_{3}\right)\right\} \\
& +b \max \left\{d\left(x_{0}, x_{1}\right), d\left(x_{0}, x_{1}\right)\right\}+c d\left(x_{1}, x_{3}\right) \\
\leq & a \max \left\{d\left(x_{0}, x_{1}\right), \frac{1}{2} d\left(x_{1}, x_{3}\right)\right\} \\
& +b d\left(x_{0}, x_{1}\right),+c d\left(x_{1}, x_{3}\right) .
\end{aligned}
$$

Applying (9) again and using (10) we have

$$
\begin{aligned}
d\left(x_{1}, x_{3}\right)= & D\left(\left\{x_{1}\right\},\left\{x_{3}\right\}\right) \\
\leq & a \max \left\{d\left(g\left(x_{0}\right), g\left(x_{1}\right)\right), d\left(g\left(x_{2}\right), g\left(x_{3}\right)\right), d\left(g\left(x_{0}\right), g\left(x_{2}\right)\right),\right. \\
& \left.\frac{1}{2}\left[d\left(g\left(x_{0}\right), g\left(x_{3}\right)\right)+d\left(g\left(x_{2}\right), g\left(x_{1}\right)\right)\right]\right\} \\
+ & b \max \left\{d\left(g\left(x_{0}\right), g\left(x_{1}\right)\right), d\left(g\left(x_{2}\right), g\left(x_{3}\right)\right)\right\} \\
+ & c\left[d\left(g\left(x_{0}\right), g\left(x_{3}\right)\right)+d\left(g\left(x_{2}\right), g\left(x_{1}\right)\right)\right] \\
\leq & a \max \left\{d\left(x_{0}, x_{1}\right), d\left(x_{2}, x_{3}\right),\left[d\left(x_{0}, x_{1}\right)+d\left(x_{1}, x_{2}\right)\right]\right. \\
& \left.\frac{1}{2}\left[d\left(x_{0}, x_{1}\right)+d\left(x_{1}, x_{2}\right)+d\left(x_{2}, x_{3}\right)+d\left(x_{2}, x_{1}\right)\right]\right\} \\
& +b \max \left\{d\left(x_{0}, x_{1}\right), d\left(x_{2}, x_{3}\right)\right\} \\
& +c\left[d\left(x_{0}, x_{1}\right)+d\left(x_{1}, x_{2}\right)+d\left(x_{2}, x_{3}\right)+d\left(x_{2}, x_{1}\right)\right] \\
\leq & (2 a+b+4 c) d\left(x_{0}, x_{1}\right) \\
= & (2-b) d\left(x_{0}, x_{1}\right) .
\end{aligned}
$$

Using (11) and (12), we get

$$
\begin{aligned}
d\left(x_{2}, x_{3}\right) \leq & a \max \left\{d\left(x_{0}, x_{1}\right), \frac{1}{2}\left[(2-b) d\left(x_{0}, x_{1}\right)\right]\right\} \\
& +b d\left(x_{0}, x_{1}\right)+c(2-b) d\left(x_{0}, x_{1}\right) \\
\leq & (1-b c) d\left(x_{0}, x_{1}\right) .
\end{aligned}
$$

It is easy to show that,

$$
d\left(x_{n+1}, x_{n}\right) \leq(1-b c)^{[n / 2]} d\left(x_{0}, x_{1}\right),
$$

where $[n / 2]$ means the greatest integer not exceeding $n / 2$. Since $b c<1$, $\left\{x_{n}\right\}$ is a Cauchy sequence and hence the sequence $\left\{x_{n}\right\}$ converges to the limit $p$ (say). 
Let $F_{m}$ be an arbitrary member of $\left\{F_{i}\right\}$. Since $\left\{x_{n}\right\} \subset F_{m}\left(x_{n-1}\right)$, by Lemma 1 , there exists a $v_{n} \in X$ such that $\left\{v_{n}\right\} \subset F_{m}(p)$ for all $n$.

Applying (9) again and using (10) we have

$$
\begin{aligned}
d\left(x_{n}, v_{n}\right)=D\left(\left\{x_{n}\right\},\left\{v_{n}\right\}\right) \leq & a \max \left\{d\left(x_{n-1}, x_{n}\right), d\left(p, v_{n}\right), d\left(x_{n-1}, p\right)\right. \\
& \left.\frac{1}{2}\left[d\left(x_{n-1}, v_{n}\right)+d\left(x_{p}, x_{n}\right)\right]\right\} \\
+ & b \max \left\{d\left(x_{n-1}, x_{n}\right), d\left(p, v_{n}\right)\right\} \\
+ & c\left[d\left(x_{n-1}, v_{n}\right)+d\left(x_{p}, x_{n}\right)\right]
\end{aligned}
$$

If $\lim _{n \rightarrow \infty} v_{n} \neq p$, then letting $n$ tend to infinity, we have

$$
\begin{aligned}
d\left(p, v_{n}\right) \leq & \text { taking a } \max \left\{d(p, p), d\left(p, v_{n}\right), d(p, p),\right. \\
& \left.\frac{1}{2}\left[d\left(p, v_{n}\right)+d(p, p)\right]\right\} \\
& +b \max \left\{d(p, p), d\left(p, v_{n}\right)\right\} \\
& +c\left[d\left(p, v_{n}\right)+d(p, p)\right] \\
\leq & (a+b+c) d\left(p, v_{n}\right) \\
< & d\left(p, v_{n}\right)
\end{aligned}
$$

a contradiction. Hence $\lim v_{n}=p$.

Since $F_{m}$ is arbitrary, then $\{p\} \subset \bigcap_{i=1}^{n} F_{i}(p)$.

\section{REFERENCES}

[1] Lj.B. Ciric, On some non-expansive type mapping and fixed points, Indian J. Pure Appl. Math., 24(3) (1993), 145-149.

[2] Lj.B. Ciric, Coincidences and fixed points of non-expansive type multi-valued and single-valued maps, Indian J. Pure Appl. Math., 26(5) (1995), 393-401.

[3] S. Heilpern, Fuzzy maps and fixed theorem, J. Math. Anal. Appl., 83 (1981), 566-569.

[4] B.S. Lee, Generalized common fixed point theorems for a sequence of fuzzy mappings, Internat. J. Math. \& Math. Sci., 17 (1994), 437-440.

[5] B.E. Rhoades, Fixed points of some Fuzzy mappings, Soochow J. Math., 22 (1996), 111-115.

[6] B.E. Rhoades, A common fixed point theorem for a sequence of fuzzy mappings, Internat. J. Math. and Math. Sci., 3 (1995), 447-450.

[7] B. Watson and B.E. Rhoades, Generalized contraction and fixed points in metric space, Math. Japonica, 34 (1989), 975-982.

[8] B. Watson and B.E. Rhoades, Fixed point for set valued mappings in metric space, Math. Japonica, 35 (1990), 735-743.

[9] L.A. Zadeh, Fuzzy sets Inform and Control, 8 (1965), 338-353. 


\section{Sweetee Mishra}

Department of Mathematics and Statistics

Dr. H.S. Gour Vishwavidyalaya (Central University) SAGAR (M.P.)

INDIA

E-mail address: sweetee_mishra@rediffmail.com

\section{R.K. NAMDEO}

H.O.D. of Mathematics and Statistics

Dr. H.S. Gour Vishwavidyalaya (Central University) SAGAR (M.P.)

INDIA

\section{Brian Fisher}

Department of Mathematics

UNIVERSITY OF LEICESTER

LEICESTER, LE1 7RH

U.K.

E-mail address: fbr@le.ac.uk 